\title{
The Tomato Hybrid Proline-rich Protein regulates the abscission zone competence to respond to ethylene signals
}

Srivignesh Sundaresan 1,2,6, Sonia Philosoph-Hadas' ${ }^{1}$, Chao Ma ${ }^{3,7}$, Cai-Zhong Jiang $\mathbb{1}^{3,4}$, Joseph Riov², Raja Mugasimangalam ${ }^{5}$, Betina Kochanek', Shoshana Salim', Michael S. Reid ${ }^{3}$ and Shimon Meir ${ }^{1}$

\begin{abstract}
The Tomato Hybrid Proline-rich Protein (THyPRP) gene was specifically expressed in the tomato (Solanum lycopersicum) flower abscission zone (FAZ), and its stable antisense silencing under the control of an abscission zone (AZ)-specific promoter, Tomato Abscission Polygalacturonase4, significantly inhibited tomato pedicel abscission following flower removal. For understanding the THyPRP role in regulating pedicel abscission, a transcriptomic analysis of the FAZ of THyPRP-silenced plants was performed, using a newly developed AZ-specific tomato microarray chip. Decreased expression of THyPRP in the silenced plants was already observed before abscission induction, resulting in FAZ-specific altered gene expression of transcription factors, epigenetic modifiers, post-translational regulators, and transporters. Our data demonstrate that the effect of THyPRP silencing on pedicel abscission was not mediated by its effect on auxin balance, but by decreased ethylene biosynthesis and response. Additionally, THyPRP silencing revealed new players, which were demonstrated for the first time to be involved in regulating pedicel abscission processes. These include: gibberellin perception, $\mathrm{Ca}^{2+}$-Calmodulin signaling, Serpins and Small Ubiquitin-related Modifier proteins involved in post-translational modifications, Synthaxin and SNARE-like proteins, which participate in exocytosis, a process necessary for cell separation. These changes, occurring in the silenced plants early after flower removal, inhibited and/ or delayed the acquisition of the competence of the FAZ cells to respond to ethylene signaling. Our results suggest that THyPRP acts as a master regulator of flower abscission in tomato, predominantly by playing a role in the regulation of the FAZ cell competence to respond to ethylene signals.
\end{abstract}

\section{Introduction}

Abscission is a natural process of plant development, in which subtended organs, leaves, flowers, fruits, and branches, separate from the parent plant ${ }^{1,2}$. The abscission process usually occurs in four phases $(\mathrm{A}-\mathrm{D})^{3-6}$. A, differentiation of undifferentiated cells to an anatomically discrete abscission zone (AZ); B, acquisition of the competence of the $\mathrm{AZ}$ cells to respond to abscission signals; $\mathbf{C}$, activation

\footnotetext{
Correspondence: Shimon Meir (shimonm@volcani.agri.gov.il)

${ }^{1}$ Department of Postharvest Science of Fresh Produce, Agricultural Research

Organization (ARO), The Volcani Center, Rishon LeZiyon, Israel

${ }^{2}$ The Robert H. Smith Institute of Plant Sciences and Genetics in Agriculture,

The Robert H. Smith Faculty of Agriculture, Food and Environment, The

Hebrew University of Jerusalem, Rehovot, Israel

Full list of author information is available at the end of the article
}

of the AZ cells by the abscission signals (mainly ethylene), and synthesis of cell wall hydrolyzing enzymes, leading to organ separation (execution phase); D, trans-differentiation of the retained portion of the $\mathrm{AZ}$ to produce a protective defense layer. Much is already known about the anatomical events of phase $\mathrm{A}$, and the physiology, biochemistry, and molecular basis of phase $\mathrm{C}^{7-10}$. The role of auxin depletion as a key event in the acquisition of the competence to respond to ethylene signaling in the AZ cells was recently reviewed $^{11}$, and we are beginning to get an insight into the above events at the molecular level ${ }^{12-18}$.

Tomato (Solanum lycopersicum) is a convenient model system to study the abscission process, since tomato plants develop a distinct AZ in the midpoint of the flower 
pedicel, referred to as flower AZ (FAZ). In the first transcriptome microarray analysis of the tomato FAZ performed following abscission induction by auxin depletion, several genes were specifically expressed in the FAZ and not in the pedicel non-AZ (NAZ) region, including KNOTTED1-LIKE HOMEOBOX PROTEIN1 (KD1), and TOMATO PROLINE RICH PROTEIN (TPRP$F 1)^{15}$. A role of KD1 in tomato flower abscission was reported $^{19}$, and the present study describes an attempt to elucidate the functional role of TPRP-F1 in abscission.

The TPRP-F1 gene, first isolated from young tomato fruit, represents a single-copy gene in the tomato genome $^{20}$. Sequence analysis of the deduced open reading frame of this gene revealed the existence of proline-rich repeated amino acid motifs ${ }^{21}$, but ignored the C-terminal domain that contains an eight-cysteine motif. Unlike this structure, the Hybrid Proline Rich Proteins (HyPRPs), which create a subgroup of structural cell wall proteins rich in proline ${ }^{22}$, are composed of a hydrophobic and two distinct domains: a proline-rich and a C-terminal domain. Regarding these findings, we refer to the tomato PRP-F1 protein in the present study as the Tomato HyPRP (THyPRP).

Protein domains binding proline-rich motifs are frequently involved in signaling events. The unique properties of proline provide a high discriminatory recognition without requiring high affinities, and therefore the structural features of proline-rich motif binding and specific recognition were investigated ${ }^{23,24}$. However, although HyPRPs are ubiquitous in plants, little is known about their roles other than the function as cell wall structural proteins ${ }^{25-27}$. Several reports indicated that subgroups of HyPRPs might have variable functions during specific developmental stages, and in response to biotic and abiotic stresses ${ }^{28-30}$. Ectopic expression of HyPRP in plants accelerated cell death, led to developmental abnormality with downregulation of reactive oxygen species-scavenging genes, and enhanced the susceptibility to pathogens by suppressing expression of defense-related genes ${ }^{31}$. Recent findings showed that another tomato HyPRP1 gene (solyc12g009650) is a negative regulator of salt and oxidative stresses, and is probably involved in sulfite metabolism ${ }^{32}$.

The regulation of the FAZ and the tomato fruit to respond to ethylene involves a cross-talk between auxin and ethylene, as auxin depletion is a prerequisite for acquiring the competence for ripening or abscission induction by ethylene ${ }^{11}$. Thus, THyPRP, which was primarily expressed in immature green tomato fruit that are ethylene insensitive, was significantly downregulated upon transition to mature green fruit that ripen in response to ethylene ${ }^{33}$. Similarly, following flower removal, a significant decreased $T H y P R P$ expression in the tomato FAZ was obtained, which was not affected by the ethylene antagonist 1-methylcyclopropene (1-MCP $)^{15}$. This indicates that the decreased THyPRP expression is a direct effect of auxin depletion.

The properties of THyPRP and its specific gene expression pattern in the tomato $\mathrm{FAZ}^{15}$ suggest, that ThyPRP has an important role in regulating the tissue competence to respond to ethylene signals. In the present report, we investigated the role of THyPRP in regulating tomato pedicel abscission induced by flower removal. For this purpose, we studied the effects of silencing the THyPRP gene under the control of the AZspecific promoter, Tomato Abscission Polygalacturonase4 (TAPG4), and performed a transcriptomic analysis of the FAZ in the wild type (WT) and the THyPRP-silenced plants.

\section{Materials and methods}

\section{Plant material and abscission induction treatments}

Tomato (Solanum lycopersicum, cv. New Yorker) seeds were obtained from the Tomato Genetics Resource Center, University of California, Davis, USA. The inflorescences of both the WT and the transgenic lines were harvested from 4-month-old greenhouse-grown plants between 08:00 and 10:00 a.m. Preparation of flower bunches, flower removal, experimental conditions, and pedicel abscission assays were performed as described before $^{15,34}$. Pedicel abscission was evaluated by careful touching the distal side of the FAZ, and monitoring the abscised pedicels for calculating the percent of pedicel abscission. For each line, we used 10-12 plants, and the experiments were repeated independently three times with similar results.

\section{Vector construction and plant transformation}

To generate TAPG4::antisense THyPRP transgenic plants, a 2379-bp fragment of the AZ-specific TAPG4 promoter from tomato genomic DNA and a 227-bp fragment of the THyPRP gene from tomato cDNA were amplified, subcloned into the modified binary vector GSA1285 in an antisense orientation, and introduced into Agrobacterium tumefaciens (LBA4404) by electroporation $^{19}$. Tomato was transformed by the tissue culture method as previously described ${ }^{35}$. Six independent transgenic lines were generated, and two representative transgenic lines (Lines 7 and 11) were selected for further analysis because they showed delayed pedicel abscission during $20 \mathrm{~h}$ after flower removal (Supplementary Figure S1). Line 5, which also showed a significantly delayed abscission, was not selected because it exhibited some additional morphological alterations (data not shown).

\section{Gene expression profiling using the Agilent platform}

The plant samples of individual time points $(0,4,8,12$, 16 , and $20 \mathrm{~h}$ after flower removal) from FAZ and NAZ of 
the WT, and FAZ of TAPG4::antisense THyPRP line 7, were obtained from two different biological experiments displaced in time, and used for the gene expression and microarray studies. The RNA was isolated and processed as previously described ${ }^{34}$. Basically, the tissue samples (50 $\mathrm{mg}$ ) were snap frozen and homogenized using a TOMY homogenizer and steel beads (TOMY Micro Smash MS100, Tomy Medico Ltd., Tokyo, Japan). RNA was isolated by Qiagen RNeasy Plant mini kit (Qiagen, Hilden, Germany) according to the manufacturer's protocol. In column DNase digestion was performed according to the protocol. The eluted RNA was stored at $-70^{\circ} \mathrm{C}$ until further processing. Total RNA purity was assessed using a NanoDrop $^{\circledR}$ ND-1000 UV-Vis spectrophotometer (Nanodrop technologies, Rockland, DE, USA). Total RNA integrity was analyzed using RNA 6000 Nano Lab Chip on the 2100 Bioanalyzer (Agilent, Palo Alto, CA, USA) following the manufacturer's protocol. A good quality RNA was defined based on the rRNA 28S/18S ratios and RNA integrity number $>6.5$.

\section{Microarray labeling, hybridization, and scanning}

Samples for gene expression analysis were labeled using Agilent Quick-Amp labeling Kit one-color. Aliquots of $500 \mathrm{ng}$ of each sample were incubated with a reverse transcription mixture at $40^{\circ} \mathrm{C}$ and converted to double stranded cDNA primed by oligo (dT) with a T7 polymerase promoter. Synthesized double stranded cDNA was used as template for cRNA generation. The cRNA was generated by in vitro transcription, and the dye Cy3 CTP (Agilent) was incorporated during this step, both carried out at $40^{\circ} \mathrm{C}$. Labeled cRNA was cleaned up, and its quality was assessed using NanoDrop ${ }^{\circledR}$ ND-1000 UVVis spectrophotometer. The specific activity determination was based on the cRNA concentration and dye incorporation.

The labeled cRNA samples were hybridized onto an AZ-specific microarray chip, AMADID: $043310^{34}$ designed by Genotypic Technology Pvt. Ltd (Bangalore, India). Aliquots of $1650 \mathrm{ng}$ of Cy3-labeled samples were fragmented and hybridized by using the Gene Expression Hybridization kit of Agilent. Hybridization was carried out in an Agilent SureHyb chamber at $65^{\circ} \mathrm{C}$ for $16 \mathrm{~h}$. The hybridized slides were washed using Agilent Gene Expression wash buffers, and scanned using the Agilent Scanner, Part Number G2600D. Data extraction from images was performed by using Feature Extraction software of Agilent V-11.5.

\section{Microarray data analysis}

Feature extracted data were analyzed using Agilent GeneSpring GX Version 12 software. Normalization of the data was done in GeneSpring GX using the 75th Percentile shift. Significant genes that were upregulated and downregulated within the group of samples were identified. Statistical $t$-test $p$-values were calculated based on volcano plot using algorithm, which allows visualization of the relationship between fold-change and statistical significance (considering both the magnitude of change and variability). Differentially regulated genes were classified based on gene ontology functional category using GeneSpring GX Analysis software. Duplicate samples were analyzed for each time point. Duplicated probes within the array were averaged for each given transcript.

\section{Gene expression validation by quantitative PCR}

Primers for qPCR were designed using the Gene Runner version 3.05 (Hastings Software Inc. Hastings, USA; http://www.generunner.net). The primers were designed to match the microarray probes, validated, and amplicon sizes were confirmed using $2 \%$ agarose gel (Supplementary Table S1). The same RNA samples were used for qPCR and microarray analysis, as previously described ${ }^{34}$. The relative expression levels of the genes were determined after normalization with ACTIN as the reference gene, using the comparative $C_{T}$ method for calculating the value of $2^{-\Delta \Delta C} \mathrm{~T}$.

\section{Sequence deposition}

The microarray data for the WT FAZ and NAZ (12 arrays each) samples were submitted under Gene Expression Omnibus database (NCBI-GEO) under the accession id GSE64221. The data for the TAPG::antisense THyPRP FAZ samples (12 arrays) were submitted under the NCBI-GEO accession id GSE64606. The data will be released for public access upon acceptance of the manuscript.

\section{Results and discussion \\ Inhibition of abscission by THyPRP silencing}

We generated 12 transgenic lines in which the THyPRP antisense construct was driven by the AZ-specific promoter, TAPG4, that controls abscission ${ }^{36}$. For the abscission experiments, we focused on two lines, 7 and 11, of the TAPG4::antisense THyPRP transgenic plants, that showed significant delayed pedicel abscission phenotypes (Supplementary Figure S1). Line 11 was also used for transcriptome analysis. The THyPRP expression in the WT was downregulated after flower removal (Fig. 1), as previously reported ${ }^{15}$. The transcript abundance was significantly lower in the THyPRP-silenced lines than in the WT, and this was manifested already before removal of open flowers, suggesting that the TAPG4 promoter was active in the FAZ of open flowers. The significantly reduced expression of THyPRP in the transgenic line 11 lasted up to $12 \mathrm{~h}$ after flower removal, during which the expression in the WT was gradually reduced as well (Fig. 1). 


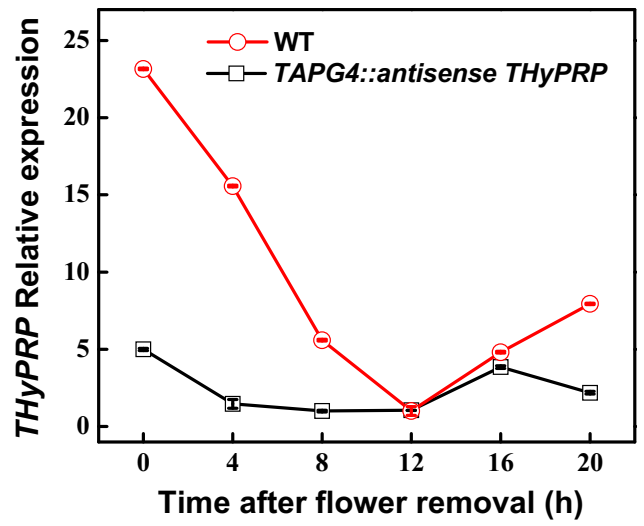

Fig. 1 Effect of antisense silencing of THyPRP (ID-X57076/ Solyc07g043000) on the kinetics of changes in qPCR expression of THyPRP in the FAZ at various time points after flower removal. qRT-PCR analysis of THyPRP expression in the FAZ of antisense transgenic line 11/generation T4 was compared to that in the WT at 0 , $4,8,12,16$, and $20 \mathrm{~h}$ after flower removal. The relative quantification of the gene expression level in the GPCR assay was determined by the comparative $C_{T}$ method $2^{-\triangle \triangle C T 95}$, using ACTIN as the reference gene. The data represent the mean values $( \pm \mathrm{SE}$ ) of duplicate experiments, each having three independent biological samples

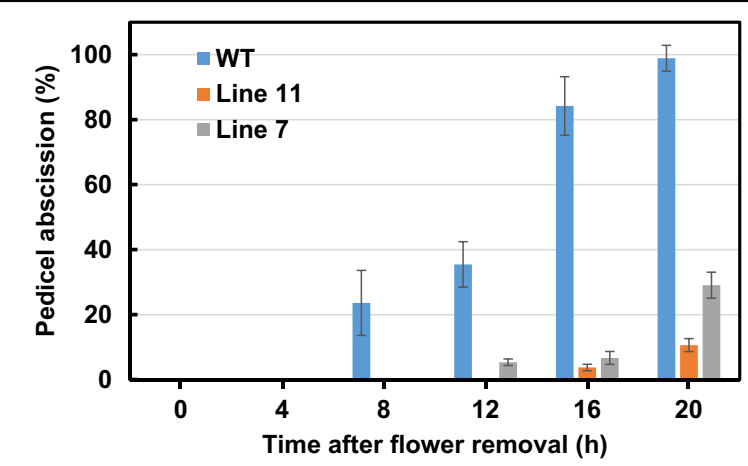

Fig. 2 Effect of antisense silencing of THyPRP on the kinetics of flower pedicel abscission following flower removal. Flower explants were prepared and handled as previously described ${ }^{15,34}$. Wild type (WT) plants (CV. NY) and silenced lines 7 and 11/generation T4 were used. The percentage of accumulated pedicel abscission was monitored at $0,4,8,12,16$, and $20 \mathrm{~h}$ after flower removal. The results are means of four replicates ( $n=30$ explants) \pm SE

Stable silencing of the THyPRP gene under the control of an AZ-specific promoter (TAPG4::antisense THyPRP) significantly inhibited pedicel abscission during $20 \mathrm{~h}$ after flower removal (Fig. 2). This indicates that the efficient silencing of the $T H y P R P$ gene affected a significant regulatory function in the FAZ, which is important for organ abscission.

\section{Transcriptomic analysis of the FAZ of the THyPRP-silenced plants}

The role of THyPRP in the regulation of abscission in the tomato flower model system was studied by
Table 1 Transcriptome responses of the FAZ tissues at various time points after flower removal in the transgenic (TAPG4::antisense THyPRP) line compared to the WT

\begin{tabular}{lll} 
Time after flower removal (h) & $\begin{array}{l}\text { Number of differentially } \\
\text { expressed transcripts in TAPG:: } \\
\text { antisense THyPRP FAZ vs. WT FAZ }\end{array}$ \\
\cline { 2 - 3 } & Upregulated & Downregulated \\
\hline 0 & 157 & 50 \\
4 & 101 & 192 \\
8 & 162 & 573 \\
12 & 141 & 296 \\
16 & 504 & 544 \\
20 & 450 & 193 \\
\hline
\end{tabular}

The total numbers of differentially expressed transcripts (fold changes: downregulated $\leq-2$; upregulated $\geq 2$ ) at different time points during the abscission process are presented.

performing a transcriptomic analysis, using an AZspecific tomato microarray chip ${ }^{34}$. The results presented in Table 1 show that 157 genes were upregulated and 50 genes were downregulated at zero time in the transgenic plants (Supplementary Table S2). It is noteworthy that changes in the expression of many of the genes that occurred between 4 and $20 \mathrm{~h}$ after flower removal were detected at more than one time point (see below). This means that the total number of modified genes $(\log 2$ based) was much lower than the sum of the modified genes listed in each time point in Table 1.

The microarray results were confirmed by two methods: (a) qPCR analyses for five selected genes (Supplementary Figure S2); (b) comparison of the abscission-related WT FAZ genes, such as genes associated with ethylene, cell wall degrading enzymes, and programmed cell death (PCD), to previous tomato FAZ microarray data ${ }^{15}$. The qPCR analyses successfully validated the microarray expression data of selected genes in all the samples from the WT and THyPRP-silenced lines (Supplementary Figure S2). We used the downregulation of cell wall modifying genes (Supplementary Figure S3), and of the Ribonuclease $T 2(L X)$ gene (Supplementary Figure S4), which were specifically upregulated in the WT FAZ following flower removal, to confirm the effect of THyPRP silencing in retarding pedicel abscission.

Enzymes associated with disassembly and modification of the cell wall include polyglacturonases (PGs), cellulases, endoglucanases, pectin methylesterases, pectate lyases, xyloglucan endotranglucosylase/hydrolases, and expansins $^{4,5,13,37,38}$. The silencing of the THyPRP gene resulted in inhibition of 28 genes encoding these cell wall modifying enzymes that were specifically upregulated in the WT following flower removal (Supplementary 


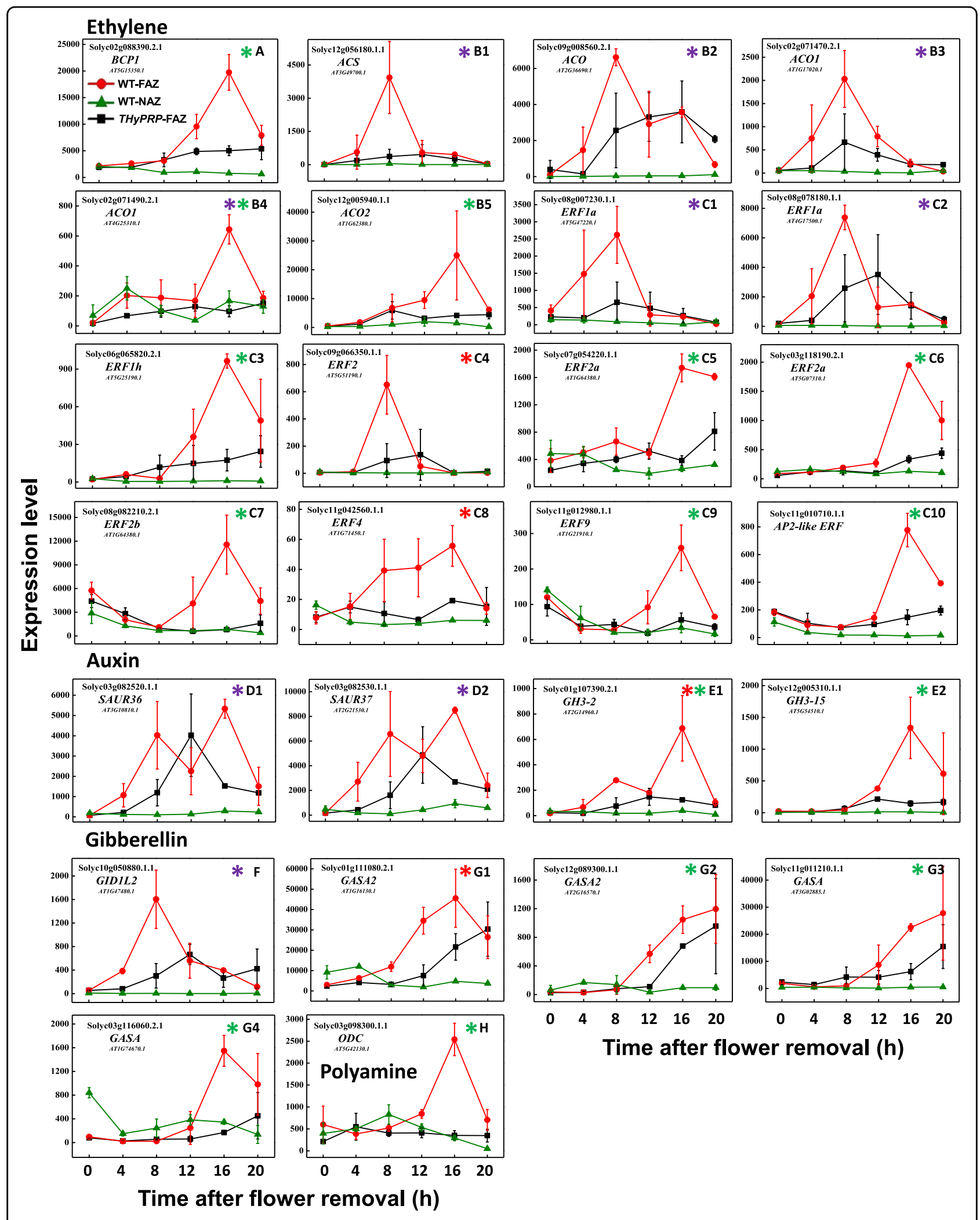

Fig. 3 (See legend on next page.) 
Fig. 3 Effect of antisense silencing of THyPRP on the kinetics of changes in array-measured expression levels of genes related to the plant hormones. Ethylene $(\mathbf{a}-\mathbf{c})$, Auxin $(\mathbf{d}, \mathbf{e})$, and Gibberellin $(\mathbf{f}, \mathbf{g})$, and Polyamine $(\mathbf{h})$, that were specifically upregulated in the WT FAZ at various time points after flower removal: early-4 $\mathrm{h}$ (blue*) or $8 \mathrm{~h}\left(\mathrm{red}^{*}\right)$, or late 12-20 h (green*).TAPG4::antisense THyPRP-silenced line 11/generation T4 was used. Expression levels were measured for Blue copper-like protein1 (BCP1) (a); 1-Aminocyclopropane-1-Carboxylate Synthase (ACS) (b1); 1-Aminocyclopropane-1-Carboxylate Oxidase (ACO) genes (b2-b5); Ethylene-Related Factor (ERF) TF genes (c1-c10); Small Auxin-Up RNA (SAUR) genes (d1, d2); Gretchen Hagen3 (GH3) which are Indole-3-acetic acid-amido synthetase genes (e1, e2); GA receptor (GID1L2) (f); GA-regulated protein (GASA) genes (g1g4); and Ornithine Decarboxylase (ODC) (h). Transcript identities are indicated by their gene ID and their Arabidopsis (At) gene number and/or their nucleotide accession number. The results are means of two independent biological replicates \pm SD

Figure S3, Supplementary Tables S2-S7). The inhibited upregulation of the cell wall modifying genes was manifested by both lower expression levels and delayed upregulation after flower removal (Supplementary Figure S3), which highly fitted the abscission inhibited phenotype of the transgenic plants. The expression patterns of TAPG1,2,4 in the WT FAZ (Supplementary Figure S2) were identical to those presented in a previous report ${ }^{15}$, confirming the results obtained from the AZ-specific tomato microarray chip analyses.

The THyPRP silencing also resulted in the inhibition of the induced expression of $L X$, which was specifically upregulated in the WT FAZ at $12-20 \mathrm{~h}$ after flower removal (Supplementary Figure S4, Supplementary Tables S5-S7). The $L X$ gene, previously studied in tomato abscission $^{15,39}$, was found in the group of 16 PCD-related genes that were specifically upregulated in the WT FAZ at a late stage $(12-20 \mathrm{~h})$ after flower removal ${ }^{40}$. Therefore, $L X$ can serve as a late marker gene for the abscission execution phase in the tomato system.

It is noteworthy that the inhibitory effects of THyPRP silencing on the induced expression of cell wall-related and $L X$ genes, resulted from the inhibitory effect of the silencing on pedicel abscission (Fig. 2). We assumed that the inhibition of pedicel abscission in the silenced plants was the result of the modified expression of regulatory genes before flower removal (zero time) and between 0 to $4 \mathrm{~h}$ after flower removal, when the competence of the FAZ cells to respond to ethylene is being acquired. Therefore, our transcriptomic analysis focused mainly on genes whose expression was changed at these time points. However, we cannot rule out the possibility that later regulatory events might occur also between 4 to $8 \mathrm{~h}$ after flower removal, coinciding with the execution of pedicel abscission, but it is difficult to distinguish at this stage between cause and effect of the abscission inhibition.

\section{THyPRP regulation of pedicel abscission is partially mediated by ethylene and gibberellin}

Figure 3 presents the plant hormone-related genes associated with abscission that were specifically upregulated in the WT FAZ after flower removal and inhibited by THyPRP silencing. The majority of these genes are related to ethylene biosynthesis and response. Thus, THyPRP silencing inhibited the upregulation of a $\mathrm{Cu}$ transporter involved in ethylene signaling (Fig. 3a), 5 ethylene biosynthesis genes (Fig. 3b1-b5), and 10 ethylene response factors (ERFs) genes (Fig. 3c1-c10), which occurred in the WT FAZ after flower removal. It is noteworthy that two ethylene biosynthesis genes, 1-aminocyclopropane-1-carboxylate synthase (ACS) and 1-amino-cyclopropane-1-carboxylate oxidase (ACO), were upregulated early $(4-8 \mathrm{~h})$ in the WT FAZ after flower removal. On the other hand, only two ERFs genes were upregulated early at $4 \mathrm{~h}$ and two at $8 \mathrm{~h}$, while the other six ERFs were upregulated at $12-20 \mathrm{~h}$ after flower removal (Fig. 3b1-c10, Supplementary Tables S3-S7). These findings suggest that THyPRP silencing can decrease ethylene biosynthesis and response. To the best of our knowledge, this is the first report that shows an effect of PRP on ethylene biosynthesis and ethylene response transcription factor (TF) genes (ERFs). Expression analysis during tomato fruit development indicated that the THyPRP gene might be downregulated by ethylene ${ }^{33}$. In soybean, GmPRP expression, upregulated in leaves infected with Phytophthora sojae, was affected by defense/ stress signaling molecules, including ethylene, salicylic acid, abscisic acid, and jasmonic acid (JA) ${ }^{41}$. These results provide an indirect evidence which supports our data on the inhibition of ethylene biosynthesis and response by downregulation of THyPRP expression.

The role of auxin as a key regulatory factor of the AZ competence to respond to ethylene was extensively documented, and was recently reviewed ${ }^{11}$. In the present tomato system, auxin depletion is artificially induced by flower removal, resulting in downregulation of many auxin-related genes ${ }^{11,15,19,42,43}$. Similarly, 211 auxinrelated genes were downregulated in the tomato FAZ and NAZ after flower removal ${ }^{40}$. However, unlike the ethylene-related genes, THyPRP silencing had no effect on the downregulation of auxin-related genes (data not shown). The downregulation of two early auxinresponsive genes, Small Auxin Upregulated RNA (SAUR) in the THyPRP-silenced plants at 4 and $16 \mathrm{~h}$ after flower removal (Fig. 3d1,d2), and the inhibition of the Gretchen Hagen 3 genes, which are related to indole-3- 


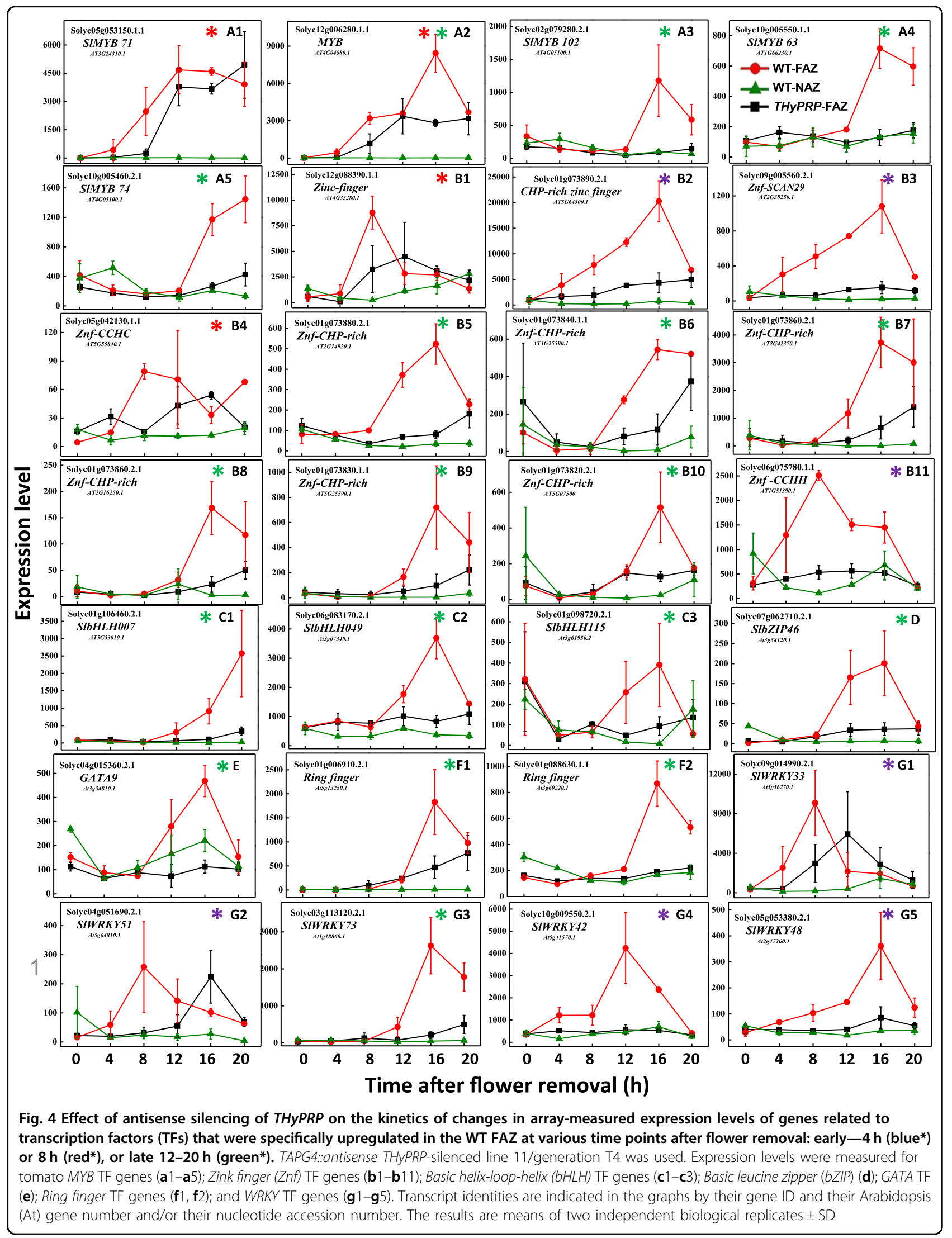


acetic acid (IAA) conjugation, at $16 \mathrm{~h}$ (Fig. 3e1,e2), do not seem to play a significant role in IAA depletion, as IAA levels are already very low at these time points ${ }^{11}$. Therefore, we rule out the possibility that the effect of THyPRP silencing on pedicel abscission is mediated by its effect on auxin balance, in contrary to the findings of tomato $K D 1$ silencing ${ }^{19}$. In the case of $K D 1$ silencing, the regulation of abscission by KD1 was associated with a changed abundance of auxin-related genes, and measurement of auxin content and activity showed that changes in KD1 expression directly modulated the auxin concentration and response in the AZ.

Interestingly, we report here for the first time that five gibberellin (GA)-related genes, upregulated specifically in the WT FAZ, were inhibited in the THyPRP-silenced plants, including the gene encoding the GA receptor, GIDIL2 (Fig. 3f) and the GA-responsive (GASA) genes (Fig. 3g1-g4, Supplementary Tables S3-S7). These results suggest that the inhibition of GIDIL2 and GASA genes by THyPRP silencing might be involved in the inhibitory effect of THYPRP silencing on tomato pedicel abscission. To the best of our knowledge, there are no published reports on the effects of GA on tomato flower abscission. The GA promoting effects on bean and cotton leaf abscission were documented long ago, and were utilized for promoting the ethylene-induced abscission of cotton leaves ${ }^{44-46}$. GA spraying at bloom is widely used as a thinning treatment for grapevine, and the molecular pathways, which regulate the acquisition of flower abscission competence following GA application to seedless Vitis vinifera, were recently reported ${ }^{47}$. Additionally, the involvement of GA genes in rose petal abscission was recently demonstrated by a transcriptome profiling of the petal $\mathrm{AZ}^{48}$.

\section{Early (0-4 h) effects of THyPRP silencing on regulatory genes}

THyPRP silencing in the FAZ at zero time altered the expression of some regulatory genes at this time point (Fig. 1). Our analysis focused on altered genes encoding functional proteins at five regulatory levels: transcriptional (homeobox and other TF genes); epigenetic; transport (peptide transporters and protein targeting, and exocytosis); post-translation related to protein degradation or phosphorylation/dephosphorylation; and signal transduction.

\section{Transcriptional regulation}

Genes related to seven TF families were specifically upregulated in the WT FAZ at different time points after flower removal, including five $M Y B$ genes (Fig. 4a1-a5), 11 Zink finger (Znf) genes (Fig. 4b1-b11), three basic helix-loop-helix (bHLH) genes (Fig. 4c1-c3), one basic leucine zipper (bZIP46) gene (Fig. 4d), one GATA9 gene
(Fig. 4e), two Ring finger genes (Fig. 4f1-F2), and five WRKY genes (Fig. 4g1-g5). The specific upregulation in the WT FAZ of all these 28 TF genes was inhibited in the THyPRP-silenced plants (Supplementary Tables S3-S7).

Three Zinc Finger (Znf) TFs, CHP-rich ZNF (Solyc01g073890.2.1) (Fig. 4b2), Znf-SCAN29 (Solyc09g005560.2.1) (Fig. 4b3), and Znf-CCHH (Solyc06g075780.1.1) (Fig. 4b11), were specifically upregulated in the WT FAZ at $4 \mathrm{~h}$ after flower removal, with a gradually increased expression up to $16 \mathrm{~h}$, which was inhibited in the THyPRP-silenced plants. The involvement of Znf genes in abscission is well documented. In the soybean leaf abscission system, 20 out of 188 (11\%) abscission-specific TF-induced genes were $Z n f s$, and most of them were induced early (at 0 and $12 \mathrm{~h}$ ) after ethylene treatment ${ }^{40}$. The Znf-ankyrin TF, which functions in a broad range of developmental processes and defense responses, was reported to play a role in the AZ establishment ${ }^{49}$. Five $Z n f$ genes were involved in calyx abscission of Korla fragrant pear ${ }^{50}$. Ten $Z n f s$ were upregulated early during induction of mature melon fruit abscission ${ }^{51}$. AtDOF4.7, a ZNF BINDING PROTEIN2 (ZFP2) gene, was initially identified within a cluster of upregulated genes in Arabidopsis stamen AZ cells before abscission ${ }^{8}$. Transgenic plants with a constitutive ZFP2 activity also exhibited delayed abscission. ZFP2 was found to interact with AtDOF4.7, suggesting that they may function together in a transcriptional complex to modulate the expression of AZ PG and other enzymes during abscission ${ }^{52}$. Our results, as well as earlier reports demonstrating a regulatory role of different $Z n f$ genes in the early stages of abscission, imply that THyPRP partly regulates abscission in tomato by mediating Znf expression.

Four WRKY TF genes, SlWRKY33 (Solyc09g014990.2.1) (Fig. 4g1), SlWRKY51 (Solyc04g051690.2.1) (Fig. 4g2), SlWRKY42 (Solyc10g009550.2.1) (Fig. 4g4), and SlWRKY48 (Solyc05g053380.2.1) (Fig. 4g5), were specifically upregulated in the WT FAZ starting at $4 \mathrm{~h}$ after flower removal, and their upregulation was inhibited in the THyPRP-silenced plants. Similarly, eight WRKY TF genes were upregulated early during mature melon fruit abscission, implying that TFs of this family might be involved in triggering the transcriptional cascade during organ abscission ${ }^{51}$. In contrast, four WRKY genes were upregulated at the late stage of ethylene-induced soybean leaf abscission ${ }^{53}$. Recently, the WRKY33 gene was found to be associated with leaf abscission in sugarcane ${ }^{32}$. WRKY33 proteins are evolutionarily conserved, having a critical role in broad stress responses, and two structural AtWRKY33 homologs were identified to function in tomato stress responses ${ }^{54}$. Additionally, the expression of the stress-induced ethylene biosynthesis genes, ACS2,6, was WRKY33-dependent ${ }^{55}$, while WRKY50,51 proteins 
mediated the signaling of the stress hormones salicylic acid and $\mathrm{JA}^{56}$. Accordingly, it is possible that the WRKY TFs are involved in tomato pedicel abscission by specifically regulating the induced expression of $A C S$ and $A C O$ genes in the FAZ (Fig. 3b1-b5).

The downregulation of THyPRP expression in the FAZ of silenced plants already at zero time before flower removal (Fig. 1) resulted in altered expression of several genes in the FAZ at this time point. Four genes were downregulated at zero time in the FAZ of the transgenic plants and remained low during $20 \mathrm{~h}$ after flower removal (Fig. 5-I): Long-chain Fatty acid CoA Ligase (Fig. 5-IA); Lipid Transfer Protein (Fig. 5-IB); High Mobility Group (HMG) Type Nucleosome Factor (Fig. 5-IC), involved in chromatin remodeling that takes part in the regulation of gene transcription at the epigenetic level; and MKIAA0930 protein (Fig. 5-ID) encoding a protein of an unknown function.

The expression of two TF genes, WUSCHEL (WUS) (Solyc02g083950.2.1) (Fig. 5-IIG) and bHLH148 (Solyc09g005070.1.1) (Fig. 5-IIH), was specifically upregulated in the FAZ of THyPRP-silenced plants at zero time. Although the expression of these genes decreased in the FAZ after flower removal, it remained significantly higher in the silenced plants than in the WT during the first $8 \mathrm{~h}$ after flower removal. WUS is a homeodomain TF produced in the cells of the niche/organizing center of the shoot apical meristems. WUS specifies stem cell fate and also restricts its own level by activating a negative regulator, CLAVATA3, in adjacent cells of the central zone $^{57}$. The tomato WUS homolog, LeWUS, was specifically expressed in the FAZ at anthesis, and was downregulated after flower removal ${ }^{17,18,58}$ and in the lines that do not differentiate AZs, JOINTLESS, and MACROCALYX-suppressed transformed plants ${ }^{58}$. This suggests that LeWUS expression in the FAZ might be involved in the regulation of the AZ development. Later studies suggested that LeWUS functions in the FAZ as a negative regulator of abscission ${ }^{17,59}$, which is in accordance with our results.

The bHLH148 gene is predicted to encode a leucinerich repeat receptor-like kinase (RLK) CLAVATA1 protein that is closely related to RLKs HAESA (HAE) and HAESALIKE2. It is well established that floral organ abscission in Arabidopsis is mediated by the small posttranslational modified peptide ligand, INFLORESCENCE DEFICIENT IN ABSCISSION, which shares a similarity in key amino acids and post-translational modifications with CLAVATA $3^{60,61}$. Our results, showing that WUS and bHLH148 (CLAVATA1) were similarly upregulated in the FAZ following THyPRP silencing (Fig. 5-IIG,IIH), suggest a possible role of THyPRP in regulating pedicel abscission in tomato.

\section{Epigenetic regulation}

The gene encoding the HMG type nucleosome/chromatin assembly factor/HMG-box DNA-binding family protein (Solyc06g050320.2), involved in the regulation of gene transcription at the epigenetic level, was specifically downregulated in the THyPRP-silenced FAZ at zero time, and exhibited a low expression level during $20 \mathrm{~h}$ after flower removal (Fig. 5-IC). Unlike this, the expression of the histone promoter-binding protein-1b(c1)-like TF gene (Solyc10g078670.1.1) was gradually upregulated in the FAZ of the transgenic plants from zero time up to $20 \mathrm{~h}$ after flower removal (Fig. 5-IIIC, Supplementary Tables S3-S7). Since the expression of these two epigenetic genes in the FAZ of the THyPRP-silenced plants was altered very early, it is suggested that this modified expression may regulate a subsequent cascade of gene transcription, thereby resulting in the inhibition of pedicel abscission. The expression of HMG proteins is highly regulated and is affected by both developmental and environmental factors ${ }^{62}$.

\section{Transport regulation-peptide transporters and protein targeting}

A peptide transporter gene, that enables the directed movement of dipeptides into, out, within, or between cells, was specifically upregulated in the FAZ of the THyPRP-silenced plants at zero time, and its expression remained high during $20 \mathrm{~h}$ after flower removal (Fig. 5-IIB). An exocytosis-related gene, Syntaxin, upregulated between 4 and $16 \mathrm{~h}$ after flower removal in the WT FAZ, was inhibited in the THyPRP-silenced plants (Fig. 6b2). Syntaxins are a family of membrane integrated Q-SNARE proteins, which primarily mediate vesicle fusion with their target membrane-bound compartments participating in exocytosis ${ }^{63}$. It is well known that mobilization of the secretory pathway should enable the release of cell wall modifying enzymes to implement abscis$\operatorname{sion}^{64,65}$. Recently, the induction of several genes involved in vesicle trafficking, such as SNARE-like protein and Syntaxin, was demonstrated in the laminar AZ of abscising citrus leaves following a cycle of water stress/ rehydration ${ }^{66}$. A sequential induction of genes encoding cell wall modifying enzymes, associated with the upregulation of genes involved in endocytosis and exocytosis during mature melon fruit abscission was reported ${ }^{51}$. Activation of vesicle trafficking involving small GTPases seems to be also required for cell wall modification during abscission of mature olive fruit ${ }^{67}$ and Arabidopsis floral $\operatorname{organs}^{68-70}$, as well as during self-pruning of spring shoots in sweet orange ${ }^{71}$. Our results suggest that THyPRP might regulate the secretion of cell wall modifying enzymes by exocytosis. 




Fig. 5 (See legend on next page.) 
Fig. 5 Line graphs showing the kinetics of changes in array-measured expression levels of genes that were specifically and continuously downregulated [I] or upregulated [II] at zero time and later on, or upregulated at 4 (blue*) or $\mathbf{8}$ (red*) h after flower removal [III] in the FAZ of THyPRP-silenced plants. TAPG4::antisense THyPRP-silenced line 11/generation T4 was used. Expression levels were measured for tomato Longchain fatty acyl-CoA synthetase (IA); Lipid transfer protein (IB); High Mobility Group (HMG) type nucleosome factor (IC); MKIAA0930 (ID); Serine protease inhibitor (Serpin) (IIA); Peptide transporter (IIB); Nucleotide Binding Site_Leucine-Rich Repeat (Cc-NBS-LRR) (IIC); Ubiquitin-like protein1 (UIp1 protease) (IID); unknown proteins (IIE1-E6); FAD-binding domain-containing protein (IIF); WUSCHEL-related homeobox-containing protein4 (WUS) (IIG); SIbHLH transcription factor148 (IIH); HTC in fruit (II-I); HAT dimerization domain-containing protein (IIJ); Nitrate transporter-TGF (IIK); MtN3-like protein (IIL); DUF599 family protein (IIM); Myrosinase-Binding protein2 (MBP2) (IIN); Plant cell wall protein SITFR88 (IIIA); Uridine 5'-diphospho (UDP)-

glucuronosyltransferase (IIIB); Transcription factor HBP-1b(c1)-like (IIIC); CONSTANS-like ZF (IIID); CONSTANS1 (CO1) TF (IIIE); Rhamnogalacturonate endolyase (IIIF); Disease resistance protein (IIIG); and defense-related Receptor-Like protein Kinase (RLK) (IIIH). Transcript identities are indicated in the graphs by their gene ID and their Arabidopsis (At) gene number and/or their nucleotide accession number. The results are means of two independent biological replicates $\pm S D$

\section{Other transporters}

Two transporter genes, nitrate transporter-TGF (Fig. 5-IIK) and Bidirectional sugar transporter SWEET12//MtN3-like protein (Fig. 5-IIL), were upregulated in the FAZ of THyPRP-silenced plants at zero time, and their expression decreased to the levels found in the WT FAZ at $4 \mathrm{~h}$ after flower removal (Supplementary Table S3). Previous reports demonstrated the upregulation of nitrate transporters genes ${ }^{15,51,67,72}$ and sugar transporters, including the gene Bidirectional sugar transporter SWEET12/MtN3-like ${ }^{15,67,73,74}$, in various AZs during organ abscission induction and execution. Our results suggest that THyPRP might regulate the expression of these transporters in the tomato FAZ.

\section{Post-translation regulation}

The importance of post-translational regulation in plants is suggested by the observations that about $10 \%$ of the Arabidopsis genome is dedicated to protein ubiquitination and phosphorylation, two of the most frequent post-translational modifications ${ }^{75,76}$. The reversible conjugation of the Small Ubiquitin-related Modifier (SUMO) peptide to protein substrates (sumoylation) is arising as a major post-translational regulatory process in all eukaryotes, including plants ${ }^{77-81}$. Components of the SUMO conjugation and deconjugation systems are conserved in plants, including tomato ${ }^{80}$.

Our results indicate for the first time the possible involvement of SERPIN or SUMO proteins in regulating abscission. Thus, genes of a serine protease inhibitor SERPIN (Solyc04g079480.2) (Fig. 5-IIA) and a ubiquitinlike protein (ULP) SUMO protease1 (Solyc08g048200.1.1) (Fig. 5-IID) were specifically upregulated in the FAZ of the THyPRP-silenced plants at zero time, and their expression remained high during $20 \mathrm{~h}$ after flower removal. In contrast, the Ulp1 protease (sumo) gene was specifically upregulated in the WT FAZ at $4 \mathrm{~h}$, peaked at $8 \mathrm{~h}$, and remained high up to $12 \mathrm{~h}$ after flower removal, while in the THyPRP-silenced plants its expression remained at a low level, similar to that in the NAZ
(Fig. 6a5, Supplementary Tables S3-S5). Each SERPIN with an inhibitory role is responsible for blocking the activity of one or more target proteins after binding, which induces an irreversible conformational change in the structure of the SERPIN, thereby disrupting its active site $^{82,83}$.

Four protein ubiquitination-related genes (F-box, $U$-box) were specifically upregulated in the WT FAZ at 4 $\mathrm{h}$, followed by a gradual increase in expression up to 8 or $16 \mathrm{~h}$ after flower removal (Fig. 6a1-a4). One of the F-box genes was completely inhibited in the transgenic plants (Fig. 6a1), while the expression of the other three was delayed, and was only partly inhibited in the THyPRPsilenced plants compared to the WT FAZ (Fig. 6a2-a4, Supplementary Tables S3-S7). Various genes related to selective ubiquitin-mediated protein degradation were specifically expressed in citrus leaf AZ after ethylene treatment ${ }^{7}$. Activity of the F-box protein HAWAIIAN SKIRT was linked to the control of petal abscission in Arabidopsis $^{84}$, and a cysteine protease $(R b C P 1)$ gene was expressed in the petal $\mathrm{AZ}$ of rose flowers ${ }^{85}$. $R b C P 1$ expression increased during natural and ethylene-induced rose petal abscission, and this increase was inhibited by 1-MCP. A mutant of CORONATINE INSENSITIVE1, an F-box protein which serves as the JA co-receptor and previously defined as Delayed Abscission4, exhibited a delayed abscission phenotype in Arabidopsis ${ }^{86}$.

Protein phosphorylation and dephosphorylated are known for decades as major mechanisms for the transmission of stress signals ${ }^{87,88}$. Our results show that genes encoding several phosphorylation/dephosphorylation enzymes, such as Serine/threonine-protein kinase receptor (Solyc04g077280.2.1) (Fig. 6h1), Phosphoenolpyruvate carboxykinase (Solyc04g076880.2.1) (Fig. 6h3), and Serine/threonine-protein phosphatase6 regulatory ankyrin repeat subunitA (Solyc06g076050.2.1) (Fig. 6h5), were specifically upregulated in the WT FAZ starting at $4 \mathrm{~h}$ after flower removal, and this upregulation was inhibited in the $T H y P R P$-silenced plants. The requirement of a MITOGEN-ACTIVATED PROTEIN kinase cascade and 


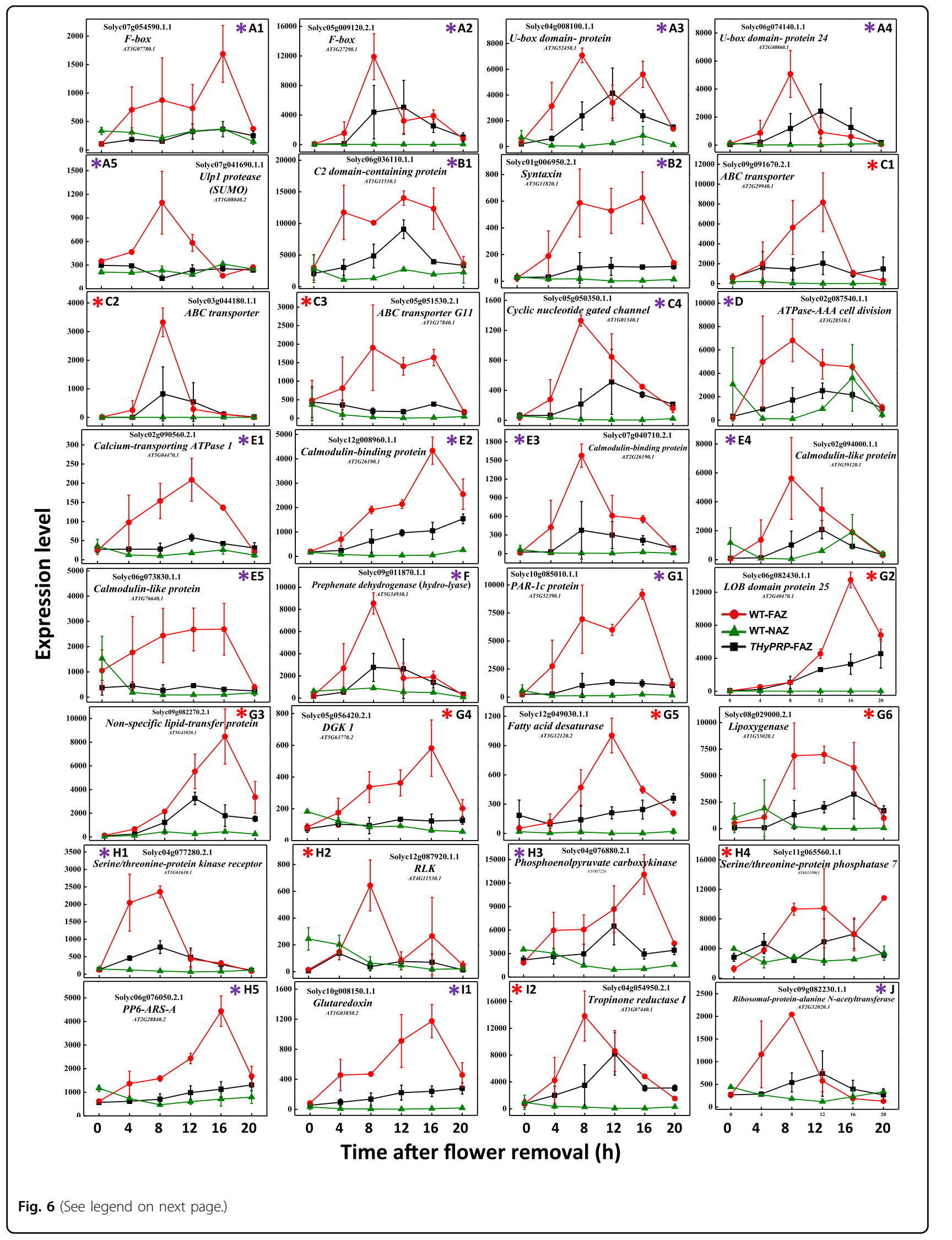


Fig. 6 Effect of antisense silencing of THyPRP on the kinetics of changes in array-measured expression levels of genes that were specifically upregulated in the WT FAZ at various time points after flower removal: early $-4 \mathrm{~h}$ (blue*) or $8 \mathrm{~h}$ (red*) or late $12-20 \mathrm{~h}$ (green*). TAPG4::antisense THyPRP silenced line 11/ generation T4 was used. Expression levels were measured for protein degradation-ubiquitin-related genes -F-box or U-box (a1-a5); genes related to transporters of macromolecules-C2 domain-containing protein (b1) and Syntaxin (b2); ABC transporter genes (c1-c3); Cyclic nucleotide-gated ion channels (c4); ATPase-AAA cell division (d); Calcium-transporting ATPase1 (e1); Calmodulin-binding protein genes (e2, e3); Calmodulin-like protein genes (e4-e5); Prephenate dehydrogenase hydrolyase (f); Lateral organ boundaries (LOB) lipids and wax-related genes - photoassimilate-responsive-1C (PAR-1C protein) (g1); LOB-domain protein25 (g2); Non-specific lipid-transfer protein (g3); Diacylglycerol kinase1 (DGK1) (g4); Fatty acid desaturase (g5); Lipoxygenase (g6); receptor kinase and protein phosphatase-related genes-Serine/threonine-protein kinase receptor (h1); Receptor-Like protein Kinase (RLK) (h2); Phosphoenolpyruvate carboxykinase (h3); Serine/threonine-protein phosphatase7 (h4); Serine/ threonine-Protein Phosphatase6 regulatory Ankyrin Repeat Subunit A (PP6-ARS-A) (h5); Redox regulation genes_Glutaredoxin (i 1 ); Tropinone reductase1 (i2); and Ribosomal-protein-alanine N-acetyl-transferase (j). Transcript identities are indicated in the graphs by their gene ID and their Arabidopsis (At) gene number, and/or their nucleotide accession number. The results are means of two biological independent replicates \pm SD

RLKs for abscission induction and execution was reported and extensively reviewed ${ }^{13,89}$. In a recent proteomic study, changes in proteins and phosphoproteins were examined in the tomato FAZ following ethylene and 1-MCP treatments ${ }^{90}$. A total of 450 phosphopeptides were detected (out of the 1429 quantified proteins), and the expression of 85 of them, corresponding to 73 phosphoproteins, was significantly modified by ethylene. These data demonstrate the unique features of the AZ phospho-proteomics, thereby suggesting the involvement of a complex network of kinase-substrate and phosphatase-substrate interactions in response to ethylene.

Another post-translational regulator gene that might be involved in abscission is ATPase-AAA cell division, which was upregulated between 4 and $16 \mathrm{~h}$ after flower removal in the WT FAZ, and its increased expression was inhibited in the THyPRP-silenced plants (Fig. 6d). The AAA modules were shown to behave as machines for folding/ unfolding polypeptides, dissociation of protein-protein interactions, and production of unidirectional motion along tracks in which a usual feature is the assembly of its subunits to hexameric or heptameric rings ${ }^{91}$. Since the ATPase-AAA was inhibited in the silenced plants, it may indicate that the cell division and post-translational functions are involved in the abscission process.

\section{Signal transduction regulation}

Our results show that seven Ca-Calmodulin $\mathrm{CCa}^{2}$ $\left.{ }^{+} / \mathrm{CaM}\right)$-related genes with a similar expression pattern were specifically upregulated between 4 and 8 or at $16 \mathrm{~h}$ after flower removal in the WT FAZ, and were inhibited in the THyPRP-silenced plants: Cyclic nucleotide-gated channel (Fig. 6c4); Calcium-transporting ATPase1 (Fig. 6e1); two CaM-binding proteins (Fig. 6e2,e3); two CaM-like proteins (Fig. 6e4,e5); and a C2 domain-containing protein (Fig. 6b1, Supplementary Tables S3-S7). C2 domain-containing proteins bind lipids and can regulate many cellular processes ${ }^{92}$. Other C2 domains were reported to act as modules for protein-protein interactions, or were suggested to play a critical role in protein localization ${ }^{93}$. These functions suggest that these proteins might be involved in the abscission process.

The $\mathrm{Ca}^{2+} / \mathrm{CaM}$ signal transduction plays a role in abscission processes. In citrus leaf abscission induced by a cycle of water stress/rehydration, a CaM gene was upregulated in the laminar AZ $1 \mathrm{~h}$ after rehydration ${ }^{66}$. During ethephon-induced litchi fruitlet abscission, 52 transcripts related to $\mathrm{Ca}^{2+}$ transport and perception displayed altered expression, among them, 19 and 33 genes were upregulated and downregulated, respectively ${ }^{94}$. Additionally, $C a M, C M L$, and Calcium-binding protein kinase genes were upregulated in the AZ during mature olive fruit abscission ${ }^{67}$. These data suggest that $\mathrm{Ca}^{2+} / \mathrm{CaM}$ signaling plays an important role in the regulatory pathways of organ abscission. Our results, demonstrating an inhibition of the upregulation of a large number of $\mathrm{Ca}^{2+} / \mathrm{CaM}$ signaling-related genes in the THyPRP-silenced plants, suggest that THyPRP plays a significant role in regulating the $\mathrm{Ca}^{2+} / \mathrm{CaM}$ signal transduction in tomato pedicel abscission.

\section{Role of THyPRP in the regulation of pedicel abscission induced by flower removal}

The aim of this study was to elucidate the possible role of THyPRP in regulating pedicel abscission induced by flower removal, by performing a transcriptome analysis of the FAZ in the THyPRP-silenced plants vs. the WT. In two previous transcriptome microarray analyses of the tomato FAZ vs. the NAZ, numerous genes were specifically expressed at zero time in the FAZ, including genes encoding TFs, hormone-related proteins, cell wallmodifying enzymes, lipid metabolism enzymes, and oth$\mathrm{ers}^{17,18}$. THyPRP (TPRP-F1) was one of the specifically expressed genes at zero time in the tomato $\mathrm{FAZ}^{15}$, and we show here that its antisense silencing significantly inhibited tomato pedicel abscission induced by flower removal (Fig. 2). 


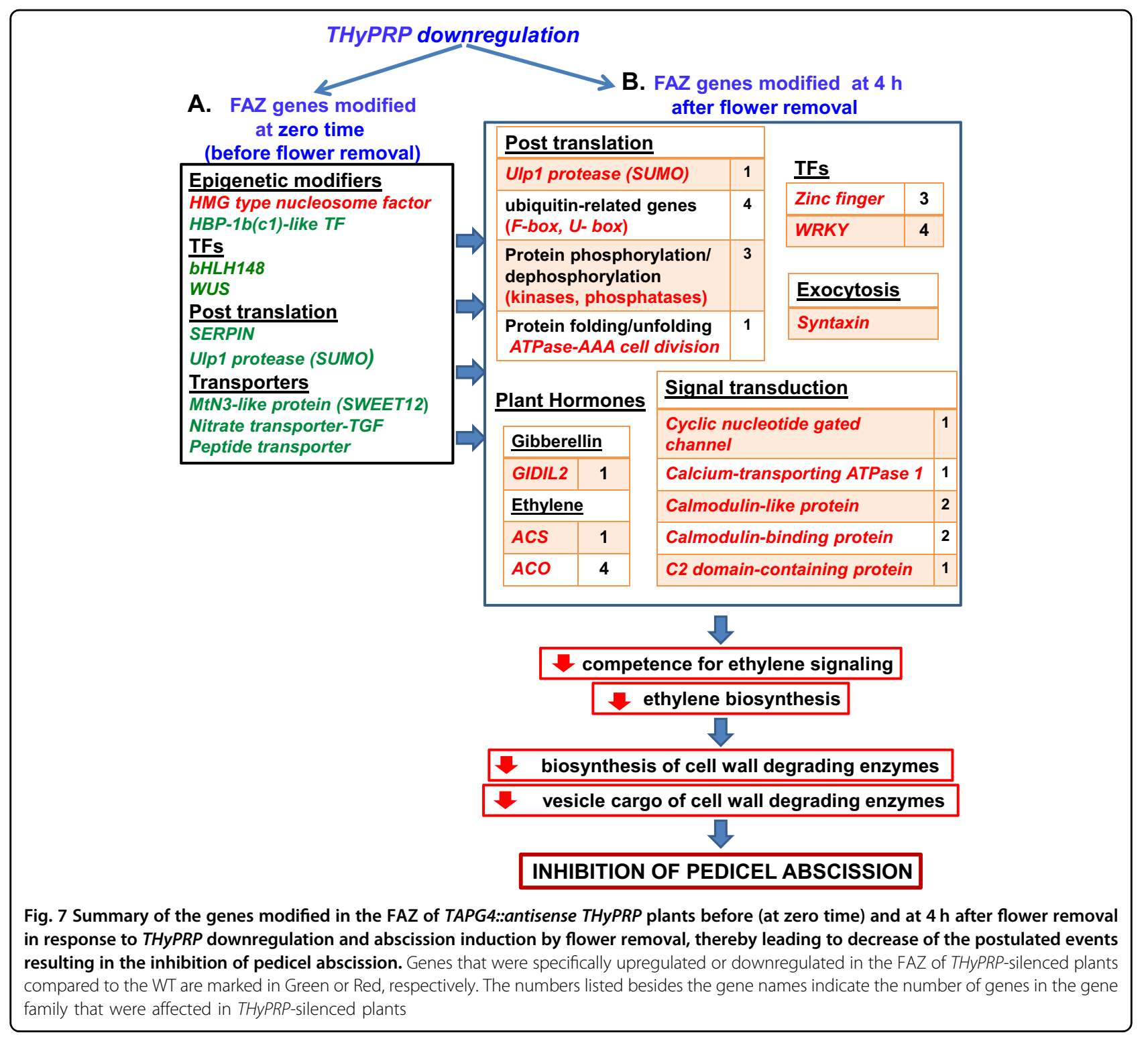

Figure 7 summarizes the postulated events leading to the inhibition of tomato pedicel abscission in the THyPRP-silenced plants. This sequence of events is based on regulatory genes whose expression was altered in the FAZ of the silenced plants at zero time and early $(0-4 \mathrm{~h})$ after flower removal, compared to their expression in the WT FAZ. The role of these regulatory genes, which are active at different regulatory levels in the abscission process, was described and discussed above.

The TAPG4 promoter was found to be a very good candidate for controlling gene silencing in the FAZ, since THyPRP expression in the silenced plants already decreased at zero time. Consequently, alternation in the expression of regulatory genes, including epigenetic modifiers, TFs, post-translational regulators, and transporters, already occurred before flower removal (Fig. 7a). We detected genes that were specifically upregulated in the WT FAZ at $4 \mathrm{~h}$ after flower removal, and their expression continued to increase later on for different periods (Fig. 7b). These upregulated genes, including genes related to ethylene biosynthesis, GA perception, TFs, post-translational regulation, and exocytosis, were inhibited in the FAZ of the THyPRP-silenced plants. Downregulation of the ethylene biosynthesis genes (ACS, ACO) in the THyPRP-silenced plants (Fig. 3b1-b5) probably leads to reduced ethylene production in the FAZ of these plants at $4 \mathrm{~h}$, and to downregulation of other genes listed in Fig. 7b, which delay and inhibit the acquisition of the competence of the FAZ cells to respond to ethylene signaling. This inhibition could have resulted 
from altered expression of regulatory genes at zero time (Fig. 7a), or from the low expression of THyPRP during the 4-h period after flower removal (Fig. 1). These two effects, resulting from the THyPRP silencing, lead in turn to downregulation of the genes involved in abscission execution (Supplementary Figures. S2, S3), finally resulting in the inhibition of the abscission phenotype (Fig. 2).

Our data suggest that the effect of THyPRP silencing on pedicel abscission was not mediated by its effect on auxin balance, but by decreased ethylene biosynthesis and response. Additionally, THyPRP silencing revealed new players, which were demonstrated for the first time to be involved in regulating pedicel abscission processes. These include: GA-perception; $\mathrm{Ca}^{2+} / \mathrm{CaM}$ signaling; SERPINS and SUMO proteins involved in post-translational modifications; Synthaxin and SNARE-like proteins, which participate in exocytosis, necessary for cell separation. Taken together, our results suggest that THyPRP is a master regulator of pedicel abscission in tomato, predominantly by playing a role in the regulation of the FAZ competence to respond to ethylene signals.

\section{Acknowledgements}

Contribution No. 778/17 from the ARO, The Volcani Center, Rishon LeZiyon, Israel. This work was supported by the United States-Israel Binational Agricultural Research and Development Fund (BARD) (grant number US-457112 C to S.M., C.-Z.J., and S.P.-H.), and the Chief Scientist of the Israeli Ministry of Agriculture Fund (grant number 203-0898-11 to S.M. and S.P-H.). Srivignesh Sundaresan would like to thank the Indian Council of Agricultural Research for providing him with an International Fellowship (ICAR-IF) to support his Ph.D. studies. We would like to acknowledge the useful assistance of Genotypic Technology Pvt., Bangalore, India, for the microarray processing and the data analysis reported in this publication.

\section{Author details}

'Department of Postharvest Science of Fresh Produce, Agricultural Research Organization (ARO), The Volcani Center, Rishon LeZiyon, Israel. ${ }^{2}$ The Robert H. Smith Institute of Plant Sciences and Genetics in Agriculture, The Robert H. Smith Faculty of Agriculture, Food and Environment, The Hebrew University of Jerusalem, Rehovot, Israel. ${ }^{3}$ Department of Plant Sciences, University of California, Davis, CA, USA. ${ }^{4}$ Crops Pathology \& Genetic Research Unit, USDAARS, Davis, CA, USA. ${ }^{5}$ Department of Bioinformatics, QTLomics Technologies Pvt. Ltd, Bangalore, India. ${ }^{6}$ Present address: Department of Nano Science and Technology, Tamil Nadu Agricultural University, Coimbatore, India. ${ }^{7}$ Present address: Beijing Key Laboratory of Development and Quality Control of Ornamental Crops, Department of Ornamental Horticulture, China Agricultural University, Beijing, China

\section{Author contributions}

Sr.S. and S.M. conceived the original screening and research plans. S.M., S.P.H., and J.R. supervised the experiments. Sr.S. performed most of the experiments and analyzed the data. C.M. and C.Z.J. prepared the constructs and produced the silenced plants. B.K. and Sh.S. provided technical assistance to Sr.S.. RM performed the microarray experiments; S.M. and S.P.H. conceived the project and wrote the article with contributions of all the authors. J.R. and M.S.R. supervised, critically revised, and complemented the writing.

\section{Conflict of interest}

The authors declare that they have no conflict of interest.

Supplementary Information accompanies this paper at https://doi.org/ 10.1038/s41438-018-0033-2.
Received: 20 December 2017 Revised: 5 March 2018 Accepted: 8 March 2018

Published online: 01 June 2018

\section{References}

1. Lewis, M. W., Leslie, M. E. \& Liljegren, S. J. Plant separation: 50 ways to leave your mother. Curr. Opin. Plant. Biol. 9, 59-65 (2006).

2. Osborne, D. J. Abscission. Crit. Rev. Plant. Sci. 8, 103-129 (1989).

3. Kim, J. Four shades of detachment: regulation of floral organ abscission. Plant Signal. Behav. 9, e976154 (2014).

4. Patterson, S. E. Cutting loose. Abscission and dehiscence in Arabidopsis. Plant. Physiol. 126, 494-500 (2001).

5. Roberts, J. A., Elliott, K. A. \& Gonzalez-Carranza, Z. H. Abscission, dehiscence, and other cell separation processes. Annu. Rev. Plant. Biol. 53, 131-158 (2002).

6. Taylor, J. E. \& Whitelaw, C. A. Signals in Abscission. New Phytol. 151, 323-340 (2001).

7. Agusti, J., Merelo, P., Cercos, M., Tadeo, F. R. \& Talon, M. Ethylene-induced differential gene expression during abscission of citrus leaves. J. Exp. Bot. 59, 2717-2733 (2008)

8. Cai, S. \& Lashbrook, C. C. Stamen abscission zone transcriptome profiling reveals new candidates for abscission control: enhanced retention of floral organs in transgenic plants overexpressing Arabidopsis ZINC FINGER PROTEIN2. Plant Physiol. 146, 1305-1321 (2008).

9. Jiang, C. Z., Lu, F., Imsabai, W., Meir, S. \& Reid, M. S. Silencing polygalacturonase expression inhibits tomato petiole abscission. J. Exp. Bot. 59, 973-979 (2008).

10. Roberts, J. A. \& Gonzalez-Carranza, Z. H. Pectinase functions in abscission. Stewart Postharvest Rev. 5, 1-4 (2009).

11. Meir, S., Sundaresan, S., Riov, J., Agarwal, I. \& Philosoph-Hadas, S. Role of auxin depletion in abscission control. Stewart Postharvest Rev. 11, 1-15 (2015).

12. Butenko, M. A. et al. Ethylene-dependent and -independent pathways controlling floral abscission are revealed to converge using promoter::reporter gene constructs in the ida abscission mutant. J. Exp. Bot. 57, 3627-3637 (2006).

13. Estornell, L. H., Agusti, J., Merelo, P., Talon, M. \& Tadeo, F. R. Elucidating mechanisms underlying organ abscission. Plant. Sci. 199-200, 48-60 (2013).

14. Meir, S., Hunter, D. A., Chen, J. C., Halaly, V. \& Reid, M. S. Molecular changes occurring during acquisition of abscission competence following auxin depletion in Mirabilis jalapa. Plant. Physiol. 141, 1604-1616 (2006).

15. Meir, S. et al. Microarray analysis of the abscission-related transcriptome in the tomato flower abscission zone in response to auxin depletion. Plant. Physiol. 154, 1929-1956 (2010).

16. Meir, S. et al. Identification of defense-related genes newly-associated with tomato flower abscission. Plant Signal. Behav. 6, 590-593 (2011).

17. Nakano, T., Fujisawa, M., Shima, Y. \& Ito, Y. Expression profiling of tomato pre-abscission pedicels provides insights into abscission zone properties including competence to respond to abscission signals. BMC Plant Biol. 13, 40 (2013).

18. Wang, X. et al. Transcriptome analysis of tomato flower pedicel tissues reveals abscission zone-specific modulation of key meristem activity genes. PLOS ONE 8, e55238 (2013).

19. $\mathrm{Ma}$, C. et al. A KNOTTED1-LIKE HOMEOBOX protein regulates abscission in tomato by modulating the auxin pathway. Plant Physiol. 167, 844-853 (2015).

20. Salts, Y., Wachs, R., Gruissem, W. \& Barg, R. Sequence coding for a nove proline-rich protein preferentially expressed in young tomato fruit. Plant Mol. Biol. 17, 149-150 (1991).

21. Salts, Y., Kenigsbuch, D., Wachs, R., Gruissem, W. \& Barg, R. DNA sequence of the tomato fruit expressed proline-rich protein gene TPRP-F1 reveals an intron within the 3 untranslated transcript. Plant Mol. Biol. 18, 407-409 (1992).

22. Jose-Estanyol, M., Gomis-Ruth, F. X. \& Puigdomenech, P. The eight-cysteine motif, a versatile structure in plant proteins. Plant Physiol. Biochem. 42, 355-365 (2004).

23. Ball, L. J., Kuhne, R., Schneider-Mergener, J. \& Oschkinat, H. Recognition of proline-rich motifs by protein-protein-interaction domains. Angew. Chem. Int Ed. Engl. 44, 2852-2869 (2005).

24. Zarrinpar, A., Bhattacharyya, R. P. \& Lim, W. A. The structure and function of proline recognition domains. Sci. Stke. 2003, Re8 (2003).

25. Jose-Estanyol, M. \& Puigdomenech, P. Plant cell wall glycoproteins and their genes. Plant Physiol. Biochem. 38, 97-108 (2000).

26. Showalter, A. M. Structure and function of plant cell wall proteins. Plant Cell. $\mathbf{5}$ 9-23 (1993). 
27. Dvorakova, L., Cvrckova, F. \& Fischer, L. Analysis of the hybrid proline-rich protein families from seven plant species suggests rapid diversification of their sequences and expression patterns. BMC Genomics 8, 412 (2007).

28. He, C. Y., Zhang, J. S. \& Chen, S. Y. A soybean gene encoding a proline-rich protein is regulated by salicylic acid, an endogenous circadian rhythm and by various stresses. Theor. Appl. Genet. 104, 1125-1131 (2002).

29. Weyman, P. D., Pan, Z., Feng, Q., Gilchrist, D. G. \& Bostock, R. M. A circadian rhythm-regulated tomato gene is induced by Arachidonic acid and Phythophthora infestans infection. Plant Physiol. 140, 235-248 (2006).

30. Zhang, Y. \& Schlappi, M. Cold responsive EARLI1 type HyPRPs improve freezing survival of yeast cells and form higher order complexes in plants. Planta 227, 233-243 (2007).

31. Yeom, S. I., Seo, E., Oh, S. K., Kim, K. W. \& Choi, D. A common plant cell-wall protein HyPRP1 has dual roles as a positive regulator of cell death and a negative regulator of basal defense against pathogens. Plant J. 69, 755-768 (2012).

32. Li, M. et al. De novo analysis of transcriptome reveals genes associated with leaf abscission in sugarcane (Saccharum officinarum L.). BMC Genomics 17, 195 (2016).

33. Santino, C. G., Stanford, G. L. \& Conner, T. W. Developmental and transgenic analysis of two tomato fruit enhanced genes. Plant Mol. Biol. 33, 405-416 (1997).

34. Sundaresan, S. et al. De novo transcriptome sequencing and development of abscission zone-specific microarray as a new molecular tool for analysis of tomato organ abscission. Front. Plant Sci. 6, 1258 (2016).

35. Fillatti, J. J., Kiser, J., Rose, R. \& Comai, L. Efficient transfer of a glyphosate tolerance gene into tomato using a binary Agrobacterium tumefaciens vector. Nat. Biotech. 5, 726-730 (1987).

36. Hong, S. B., Sexton, R. \& Tucker, M. L. Analysis of gene promoters for two tomato polygalacturonases expressed in abscission zones and the stigma. Plant. Physiol. 123, 869-881 (2000).

37. Lashbrook, C. C. Functional genomic approaches to abscission. Stewart Posthavest Rev. 5, 1-7 (2009).

38. Lashbrook, C. C. \& Cai, S. Cell wall remodeling in Arabidopsis stamen abscission zones: temporal aspects of control inferred from transcriptional profiling. Plant Signal. Behav. 3, 733-736 (2008).

39. Bar-Dror, T. et al. Programmed cell death occurs asymmetrically during abscission in tomato. Plant Cell 23, 4146-4163 (2011).

40. Kim, J. et al. Examination of the abscission-associated transcriptomes for soybean, tomato, and arabidopsis highlights the conserved biosynthesis of an extensible extracellular matrix and boundary layer. Front. Plant Sci. 6, 1109 (2015).

41. Jiang, L. et al. Isolation and characterization of a novel pathogenesis-related protein gene (Gmprp) with induced expression in soybean (Glycine max) during infection with Phytophthora sojae. PLOS ONE 10, e0129932 (2015).

42. Guan, X. et al. Temporal and spatial distribution of AUXIN RESPONSE FACTOR genes during tomato flower abscission. J. Plant Growth Regul. 33, 317-327 (2014).

43. Zuo, X. et al. Expression patterns of auxin-responsive genes during tomato flower pedicel abscission and potential effects of calcium. Aust. J. Bot. 60 , 68-78 (2012).

44. Bornman, C. H., Addicott, F. T. \& Spurr, A. R. Auxin and gibberellin effects on cell growth and starch during abscission in cotton. Plant Physiol. 41, 871-876 (1966).

45. Chatterjee, S. K. \& Leopold, A. C. Kinetin and gibberellin actions on abscission processes. Plant Physiol. 39, 334-337 (1964).

46. Morgan, P. W. \& Durham, J. I. Ethylene-induced leaf abscission is promoted by gibberellic acid. Plant Physiol. 55, 308-311 (1975).

47. Domingos, S. et al. Shared and divergent pathways for flower abscission are triggered by gibberellic acid and carbon starvation in seedless Vitis vinifera $\mathrm{L}$. BMC Plant Biol. 16, 38 (2016).

48. Gao, Y. et al. Transcriptome profiling of petal abscission zone and functional analysis of an AUX/AA family gene RhIAA16 involved in petal shedding in rose. Front. Plant Sci. 7, 1375 (2016).

49. Khan, M., Xu, H. \& Hepworth, S. R. BLADE-ON-PETIOLE genes: setting boundaries in development and defense. Plant Sci. 215-216, 157-171 (2014).

50. Pei, M., Niu, J., Li, C., Cao, F. \& Quan, S. Identification and expression analysis of genes related to calyx persistence in Korla fragrant pear. BMC Genomics 17, 132 (2016).

51. Corbacho, J., Romojaro, F., Pech, J. C., Latche, A. \& Gomez-Jimenez, M. C. Transcriptomic events involved in melon mature-fruit abscission comprise the sequential induction of cell-wall degrading genes coupled to a stimulation of endo and exocytosis. PLOS ONE 8, e58363 (2013).

52. Wei, P. C. et al. Overexpression of AtDOF4.7, an Arabidopsis DOF family transcription factor, induces floral organ abscission deficiency in Arabidopsis. Plant Physiol. 153, 1031-1045 (2010).

53. Kim, J. et al. Transcriptome analysis of soybean leaf abscission identifies transcriptional regulators of organ polarity and cell fate. Front. Plant Sci. 7, 125 (2016).

54. Zhou, J. et al. Characterization of the promoter and extended C-terminal domain of Arabidopsis WRKY33 and functional analysis of tomato WRKY33 homologues in plant stress responses. J. Exp. Bot. 66, 4567-4583 (2015).

55. Datta, R. et al. Glutathione regulates 1-aminocyclopropane-1-carboxylate synthase transcription via WRKY33 and 1-aminocyclopropane-1-carboxylate oxidase by modulating messenger RNA stability to induce ethylene synthesis during stress. Plant Physiol. 169, 2963-2981 (2015).

56. Gao, Q. M., Venugopal, S., Navarre, D. \& Kachroo, A. Low oleic acid-derived repression of jasmonic acid-inducible defense responses requires the WRKY50 and WRKY51 proteins. Plant Physiol. 155, 464-476 (2011).

57. Yadav, R. K. \& Reddy, G. V. WUSCHEL protein movement and stem cell homeostasis. Plant Signal. Behav. 7, 592-594 (2012).

58. Nakano, T. et al. MACROCALYX and JOINTLESS interact in the transcriptiona regulation of tomato fruit abscission zone development. Plant Physiol. 158, 439-450 (2012).

59. Nakano, T., Fujisawa, M., Shima, Y. \& Ito, Y. The AP2/ERF transcription factor SIERF52 functions in flower pedicel abscission in tomato. J. Exp. Bot. 65, 3111-3119 (2014).

60. Butenko, M. A. et al. INFLORESCENCE DEFICIENT IN ABSCISSION controls floral organ abscission in Arabidopsis and identifies a novel family of putative ligands in plants. Plant Cell 15, 2296-2307 (2003).

61. Stenvik, G. E. et al. The EPIP peptide of INFLORESCENCE DEFICIENT IN ABSCISSION is sufficient to induce abscission in Arabidopsis through the receptor-like kinases HAESA and HAESA-LIKE2. Plant Cell 20, 1805-1817 (2008).

62. Reeves, R. Nuclear functions of the HMG proteins. Biochim. Biophys. Acta 1799 3-14 (2010).

63. Lipka, V., Kwon, C. \& Panstruga, R. SNARE-ware: the role of SNARE-domain proteins in plant biology. Annu. Rev. Cell Dev. Biol. 23, 147-174 (2007).

64. Sexton, R. \& Hall, J. L. Fine structure and cytochemistry of the abscission zone cells of phaseolus leaves: I. Ultrastructural changes occurring during abscission. Ann. Bot. 38, 849-854 (1974).

65. Sexton, R., Jamieson, G. G. C. \& Allan, M. H. I. L. An ultrastructural study of abscission zone cells with special reference to the mechanism of enzyme secretion. Protoplasma 91, 369-387 (1977).

66. Agusti, J. et al. Early gene expression events in the laminar abscission zone of abscission-promoted citrus leaves after a cycle of water stress/rehydration: involvement of CitbHLH1. J. Exp. Bot. 63, 6079-6091 (2012).

67. Gil-Amado, J. A. \& Gomez-Jimenez, M. C. Transcriptome analysis of mature fruit abscission control in olive. Plant Cell Physiol. 54, 244-269 (2013).

68. Leslie, M. E., Lewis, M. W., Youn, J. Y., Daniels, M. J. \& Liljegren, S. J. The EVERSHED receptor-like kinase modulates floral organ shedding in Arabidopsis. Development 137, 467-476 (2010).

69. Liljegren, S. J. Organ abscission: exit strategies require signals and moving traffic. Curr. Opin. Plant Biol. 15, 670-676 (2012).

70. Liljegren, S. J. et al. Regulation of membrane trafficking and organ separation by the NEVERSHED ARF-GAP protein. Development 136, 1909-1918 (2009).

71. Zhang, J. Z., Zhao, K., Ai, X. Y. \& Hu, C. G. Involvements of PCD and changes in gene expression profile during self-pruning of spring shoots in sweet orange (Citrus sinensis). BMC Genomics 15, 892 (2014).

72. Sundaresan, S. et al. Abscission of flowers and floral organs is closely associated with alkalization of the cytosol in abscission zone cells. J. Exp. Bot. 66 1355-1368 (2015)

73. Reinders, A., Panshyshyn, J. A. \& Ward, J. M. Analysis of transport activity of Arabidopsis sugar alcohol permease homolog AtPLT5. J. Biol. Chem. 280, 1594-1602 (2005)

74. Cheng, Y.-Q. et al. RNA-seq analysis reveals ethylene-mediated reproductive organ development and abscission in soybean (Glycine max L. Merr.). Plant Mol. Biol. Report 31, 607-619 (2013).

75. Mann, M. \& Jensen, O. N. Proteomic analysis of post-translational modifications. Nat. Biotechnol. 21, 255-261 (2003).

76. Mazzucotelli, E. et al. The E3 ubiquitin ligase gene family in plants: regulation by degradation. Curr. Genomics 7, 509-522 (2006). 
77. del Pozo, J. C. \& Estelle, M. F-box proteins and protein degradation: an emerging theme in cellular regulation. Plant Mol. Biol. 44, 123-128 (2000).

78. Estelle, M. Proteases and cellular regulation in plants. Curr. Opin. Plant. Biol. 4, 254-260 (2001).

79. Vierstra, R. D. The expanding universe of ubiquitin and ubiquitin-like modifiers. Plant Physiol. 160, 2-14 (2012)

80. Miura, K. \& Hasegawa, P. M. Sumoylation and other ubiquitin-like post-translational modifications in plants. Trends Cell Biol. 20, 223-232 (2010).

81. Miura, K., Jin, J. B. \& Hasegawa, P. M. Sumoylation, a post-translational regulatory process in plants. Curr. Opin. Plant. Biol. 10, 495-502 (2007).

82. Law, R. H. et al. An overview of the serpin superfamily. Genome Biol. 7, 216 (2006).

83. Khan, M. S. et al. Serpin inhibition mechanism: a delicate balance between native metastable state and polymerization. J. Amino Acids 2011, 606797 (2011).

84. Gonzalez-Carranza, Z. H. et al. HAWAIIAN SKIRT: an F-box gene that regulates organ fusion and growth in Arabidopsis. Plant Physiol. 144, 1370-1382 (2007).

85. Tripathi, S. K., Singh, A. P., Sane, A. P. \& Nath, P. Transcriptional activation of a 37 $\mathrm{kDa}$ ethylene responsive cysteine protease gene, $R b C P 1$, is associated with protein degradation during petal abscission in rose. J. Exp. Bot. 60, 2035-2044 (2009).

86. Kim, J., Patterson, S. E. \& Binder, B. M. Reducing jasmonic acid levels causesein2 mutants to become ethylene responsive. FEBS Lett. 587, 226-230 (2013).
87. Rochaix, J. D. et al. Protein kinases and phosphatases involved in the acclimation of the photosynthetic apparatus to a changing light environment. Philos. Trans. R. Soc. Lond. B Biol. Sci. 367, 3466-3474 (2012).

88. Trewavas, A. Post-translational modification of proteins by phosphorylation. Annu. Rev. Plant. Physiol. 27, 349-374 (1976).

89. Patharkar, O. R. \& Walker, J. C. Floral organ abscission is regulated by a positive feedback loop. Proc. Natl Acad. Sci. USA 112, 2906-2911 (2015).

90. Zhang, X. L., Qi, M. F., Xu, T., Lu, X. J. \& Li, T. L. Proteomics profiling of ethyleneinduced tomato flower pedicel abscission. J. Proteom. 121, 67-87 (2015).

91. Santos, L. Molecular mechanisms of the AAA proteins in plants. Adv. Agric Food Biotechnol. 37, 1-15 (2006).

92. Lee, C. B., Swatek, K. N. \& McClure, B. Pollen proteins bind to the C-terminal domain of Nicotiana alata pistil arabinogalactan proteins. J. Biol. Chem. 283 26965-26973 (2008).

93. Kim, Y. C., Kim, S. Y., Choi, D., Ryu, C. M. \& Park, J. M. Molecular characterization of a pepper C2 domain-containing SRC2 protein implicated in resistance against host and non-host pathogens and abiotic stresses. Planta 227, 1169-1179 (2008)

94. Li, C., Wang, Y., Ying, P., Ma, W. \& Li, J. Genome-wide digital transcript analysis of putative fruitlet abscission related genes regulated by ethephon in litchi. Front. Plant Sci. 6, 502 (2015).

95. Livak, K. J. \& Schmittgen, T. D. Analysis of relative gene expression data using real-time quantitative PCR and the 2(-Delta Delta C(T)) method. Methods 25 , 402-408 (2001). 